\title{
Device Status Unknown
}

National Cancer Institute

\section{Source}

National Cancer Institute. Device Status Unknown. NCI Thesaurus. Code C160945.

The status of the device is unknown. 